\title{
Le VPF, facteur de perméabilité vasculaire
}

\section{Les nouvelles de ce numéro ont été préparées par : Pascale Briand Jean-Claude Dreyfus Axel Kahn Marc Peschanski}

\footnotetext{
* Depuis la rédaction de cette nouvelle, ce travail a été complété pur les deux groupes. La protéine humaine compte 215 acides aminés dont une séquence signal de 26 acides aminés. Elle est apparentée à la chaîne B du PDGF.

Les facteurs capables de promouvoir l'angiogenèse sont nombreux; ils ont été présentés dans médecine/sciences en 1988 ( $n^{\circ} 5$, vol. 4, p. 318). Un facteur qui stimule lui aussi l'angiogenèse, mais dont l'effet primitif semble être d'augmenter la perméabilité des vaisseaux sanguins, a été identifié en 1983 à partir du milieu conditionné d'une lignée tumorale de cobaye [1]. Tout dernièrement, les propriétés de ce facteur de perméabilité vasculaire ou VPF ont été précisées dans deux laboratoires, l'un de Saint Louis (MO, USA), à partir de cette même lignée [2], l'autre de Genentech (San Francisco, CA, USA), à partir de cellules folliculaires d'hypophyse de bœuf [3]. L'identité de ce nouvel acteur intervenant dans le système vasculaire repose sur ses propriétés chimiques et biologiques.

(1) Le poids moléculaire du VPF non dissocié est de 40-45 kDa, celui de la sous-unité de 18-24 kDa. Un trait marquant de cette protéine est sa très grande hétérogénéité, tant de taille que de charge. Cependant, le séquençage deș acides aminés de l'extrémité $\mathrm{N}$-terminale effectué par les deux groupes - 36 acides aminés [2] ou cinq acides aminés [3] - montre une séquence unique, sans homologie avec des séquences connues, et obtenue avec des isoformes différentes $^{*}$. Enfin, des anticorps dirigés contre la partie $\mathrm{N}$-terminale du VPF abolissent toutes ses activités.

(2) L'effet biologique du VPF s'adresse exclusivement aux cellules endothéliales in vivo comme in vitro. Le test classique est celui de l'injection intradermique au cobaye, montrant le passage d'un colorant, le bleu d'Evans, des vaisseaux vers les tissus, marqué par l'apparition de taches bleues aux sites d'injection; la dose efficace est de l'ordre du nanogramme. Une deuxième activité consiste en un pouvoir mitogénique sur les cellules endothéliales: le VPF déclenche, en culture, l'incorporation de thymidine puis la multiplication; il agit aussi in vivo dans le test de la cornée du rat. Mais, et ce caractère est spécifique du VPF, seules ces cellules réagissent. Il n'y a aucun effet sur une série d'autres tissus, contrairement à la plupart des autres facteurs angiogéniques.

L'interaction entre le VPF marqué à l'iode et des cellules endothéliales provenant de la veine ombilicale humaine met en évidence des récepteurs de surface spécifiques qui sont encore en cours d'étude. Enfin, comme le VPF peut être sécrété par de nombreuses cellules tumorales [4], il se pourrait qu'il joue un rôle dans la stimulation de la croissance des vaisseaux allant irriguer les tumeurs.

Le VPF possède donc des propriétés particulières qui permettent d'envisager son intervention dans la perméabilité vasculaire et la néovascularisation, mais aussi dans l'inflammation et les processus de guérison des plaies.

J.-C. D.

1. Senger DR, Galli SJ, Dvorak AM, et al. Tumor cells secrete a vascular permeability factor that promotes accumulation of ascites fluid. Science $1983 ; 219: 983-5$.

2. Connolly DT, Heuvelman DM, Nelson R, et al. Tumor vascular permeability factor stimulates endothelial cell growth and angiogenesis. J Clin Invest 1989; $84: 1470-8$.

3. Ferrara N, Henzel WJ. Pituitary follicular cells secrete a novel heparin-binding growth factor specific for vascular endothelial cells. Biochem Biophys Res Commun 1989; 161 : 851-8.

4. Senger DR, Perruzzi CA, Feder J, Dvorak HF. A highly conserved vascular permeability factor secreted by a variety of human and rodent cell lines. Cancer Res 1986 ; 46:5629-32. 5. Leung DW, Cachianes G, Kuang WJ, Goeddel DV, Ferrara N. Vascular endothelial growth factor is a secreted angiogenic mitogen. Science 1989; 246 : 1306-9.

6. Keck PJ, Hause SD, Krivi G, et al. Vascular permeability factor, an endothelial cell mitogen related to PDGF. Science 1989; $246: 1309-12$.

西

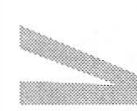

